\title{
„Kinder und Jugendliche benötigen verlässliche Bildungsangebote“"
}

\section{Deutscher Bibliotheksverband begrüßt die angekündigte Fortsetzung von „Kultur macht stark“}

https://doi.org/10.1515/bd-2021-0068

Das erfolgreiche Bundesprogramm „Kultur macht stark. Bündnisse für Bildung“ des Bundesministeriums für Bildung und Forschung (BMBF) soll ab 2023 für weitere fünf Jahre fortgesetzt werden. Dies ist ein wichtiges Signal für die beteiligten Akteur*innen, die vor Ort kulturelle Bildungsangebote für Kinder und Jugendliche umsetzen.

Der Deutsche Bibliotheksverband e.V. (dbv) begrüßt die Entscheidung, die bewährte Programmstruktur in der Zusammenarbeit mit bundesweit tätigen Verbänden und Initiativen fortzuführen, die für die Qualitätssicherung und die Vielfalt der kulturellen Bildungsangebote stehen. Gemeinsam mit den beteiligten Programmpartnern hat sich der dbv intensiv dafür eingesetzt, dass das Programm nun bis Ende 2027 verlängert wird.

Prof. Dr. Andreas Degkwitz, Bundesvorsitzender des dbv, sagte dazu: „Kinder und Jugendliche, deren Bildungszugänge erschwert sind, sind auf verlässliche Angebote außerschulischer Kultur- und Bildungseinrichtungen wie Bibliotheken angewiesen. Dafür bedarf es einer nachhaltigen Förderung. Gerade kulturelle Projekte außerhalb der Schule öffnen neue Perspektiven und stärken Kinder in ihren Entfaltungsmöglichkeiten. Sie entdecken so nichtkommerzielle Orte, die ihnen offenstehen und an denen sie kreativ sein können. Durch die pandemiebedingten Einschränkungen, unter denen besonders diese Kinder und Jugendlichen leiden, ist das Programm wichtiger denn je. Seine Finanzierung muss der neu konstituierte Deutsche Bundestag schnellstmöglich absichern.“

Damit das Programm zukünftig noch mehr Kinder und Jugendliche erreichen kann, ist es aus Sicht des dbv zentral, dass sich neben einer gesicherten Finanzierung durch schlanke Verfahren und praxisnahe Vorgaben möglichst viele Einrichtungen an dem Programm beteiligen können. Im Sinne der Zielgruppe ist zudem ein inklusiver Ansatz erforderlich, der den vielfachen Benachteiligungen gerecht wird und gleichzeitig nicht stigmatisierend wirkt. Eine bessere kommunale Verankerung fördert zudem die nachhaltige Wirksamkeit der Projekte und kann die Arbeit vor Ort erleichtern. Um Vorhaben im ländlichen Raum besser 
fördern zu können, ist es erforderlich, den dortigen Bedingungen Rechnung zu tragen.

Das Ziel des Bundesbildungsministeriums, zukünftig verstärkt digitale Zugänge zu fördern, unterstützt der dbv ausdrücklich, der sich seit 2013 mit verschiedenen Programmen zur digitalen Leseförderung bei „Kultur macht stark“ beteiligt. Das Förderprogramm wird so der Lebensrealität der Kinder und Jugendlichen gerecht und stärkt ihre kreativen Ausdrucks- und Beteiligungsmöglichkeiten im Zeitalter der Digitalität.

\section{„Total Digital! Lesen und erzählen mit digitalen Medien“}

Der dbv beteiligt sich seit 2013 am Förderprogramm „Kultur macht stark. Bündnisse für Bildung“ des Bundesministeriums für Bildung und Forschung (BMBF). In der ersten Programmphase von 2013 bis 2017 mit dem Projekt „Lesen macht stark: Lesen und digitale Medien“, seit 2018 mit dem Projekt „Total Digital!“. Das Förderprogramm richtet sich in erster Linie an Kinder und Jugendliche von drei bis achtzehn Jahren, denen der Zugang zu Bildung erschwert ist. Mit „Kultur macht stark“ fördert das BMBF auch in der zweiten Programmphase (2018 bis 2022) außerschulische Projekte der Kulturellen Bildung für mehr gesellschaftliche Teilhabe und Chancengleichheit mit 250 Millionen Euro. Neben dem dbv sind 28 weitere Verbände und Initiativen Programmpartner von „Kultur macht stark“.

Nähere Informationen zu dem Förderprogramm finden Sie unter: www.lesenund-digitale-medien.de.

\section{Der Deutsche Bibliotheksverband e.V. (dbv)}

Der Deutsche Bibliotheksverband e.V. (dbv) vertritt mit seinen mehr als 2.100 Mitgliedern bundesweit rund 10.000 Bibliotheken mit 25.000 Beschäftigten und 11 Mio. Nutzer^innen. Sein zentrales Anliegen ist es, Bibliotheken zu stärken, damit sie allen Bürger`innen freien Zugang zu Informationen ermöglichen. Der Verband setzt sich ein für die Entwicklung innovativer Bibliotheksleistungen für Wissenschaft und Gesellschaft. Als politische Interessensvertretung unterstützt der dbv die Bibliotheken insbesondere auf den Feldern Informationskompetenz und Medienbildung, Leseförderung und bei der Ermöglichung kultureller und gesellschaftlicher Teilhabe für alle Bürger*innen.

Mehr Informationen finden Sie unter www.bibliotheksverband.de. 


\section{Pressekontakt}

Kristin Bäßler

Deutscher Bibliotheksverband

Leitung Kommunikation / Pressesprecherin

Tel.: + 49 (0)30 6449899 25 | E-Mail: baessler@bibliotheksverband.de

\section{Projektkontakt}

Brigitta Wühr

Projektleiterin „Total Digital!“

Tel.: + 49 (0)30644989913 | E-Mail: wuehr@bibliotheksverband.de

URL: www.lesen-und-digitale-medien.de 